PROCEEDINGS OF THE

AMERICAN MATHEMATICAL SOCIETY

Volume 137, Number 3, March 2009, Pages 931-935

S 0002-9939(08)09592-0

Article electronically published on September 25, 2008

\title{
A NOTE ON EVALUATIONS OF MULTIPLE ZETA VALUES
}

\author{
SHUICHI MUNETA
}

(Communicated by Ken Ono)

\begin{abstract}
In this paper we give a short and simple proof of the remarkable evaluations of multiple zeta values established by D. Bowman and D. M. Bradley.
\end{abstract}

\section{INTRODUCTION}

The multiple zeta value (MZV) is defined by the convergent series

$$
\zeta\left(k_{1}, k_{2}, \ldots, k_{n}\right):=\sum_{m_{1}>m_{2}>\cdots>m_{n}>0} \frac{1}{m_{1}^{k_{1}} m_{2}^{k_{2}} \cdots m_{n}^{k_{n}}},
$$

where $k_{1}, k_{2}, \ldots, k_{n}$ are positive integers and $k_{1} \geq 2$. One remarkable property of MZVs is that MZVs are evaluated for some special arguments as rational multiples of powers of $\pi^{2}$. For example, the following evaluations were proven by many authors $([1],[5], 8])$ :

$$
\zeta\left(\{2\}_{m}\right)=\frac{\pi^{2 m}}{(2 m+1) !} \quad\left(m \in \mathbb{Z}_{>0}\right)
$$

where $\{2\}_{m}$ denotes the $m$-tuple $(2,2, \ldots, 2)$. In [8], D. Zagier conjectured the following evaluations:

$$
\zeta\left(\{3,1\}_{n}\right)=\frac{2 \pi^{4 n}}{(4 n+2) !} \quad\left(n \in \mathbb{Z}_{>0}\right) .
$$

These evaluations were proved by J. M. Borwein, D. M. Bradley, D. J. Broadhurst and P. Lisoněk ([2], 3]). In addition, D. Bowman and D. M. Bradley proved the following theorem which contained these results:

Theorem 1 ([4]). For nonnegative integers $m$, $n$, we have

$$
\begin{gathered}
\sum_{\substack{j_{0}+j_{1}+\cdots+j_{2 n}=m \\
j_{0}, j_{1}, \ldots, j_{2 n} \geq 0}} \zeta\left(\{2\}_{j_{0}}, 3,\{2\}_{j_{1}}, 1,\{2\}_{j_{2}}, \ldots,\{2\}_{j_{2 n-2}}, 3,\{2\}_{j_{2 n-1}}, 1,\{2\}_{j_{2 n}}\right) \\
=\left(\begin{array}{c}
m+2 n \\
m
\end{array}\right) \frac{\pi^{2 m+4 n}}{(2 n+1) \cdot(2 m+4 n+1) !} .
\end{gathered}
$$

In this article we provide a short and simple proof of Theorem 1 which refines the proof of Theorem 5.1 in [4].

Received by the editors March 13, 2008, and, in revised form, March 29, 2008. 2000 Mathematics Subject Classification. Primary 11M41; Secondary 11M06.

Key words and phrases. Multiple zeta values, shuffle.

(C)2008 American Mathematical Society Reverts to public domain 28 years from publication 


\section{Algebraic Setup}

We summarize the algebraic setup of MZVs introduced by Hoffman (cf. [6], 7]). Let $\mathfrak{H}=\mathbb{Q}\langle x, y\rangle$ be the noncommutative polynomial ring in two indeterminates $x, y$ and $\mathfrak{H}^{1}$ and $\mathfrak{H}^{0}$ its subrings $\mathbb{Q}+\mathfrak{H} y$ and $\mathbb{Q}+x \mathfrak{H} y$. We set $z_{k}=x^{k-1} y(k=1,2,3, \ldots)$. Then $\mathfrak{H}^{1}$ is freely generated by $\left\{z_{k}\right\}_{k \geq 1}$.

We define the $\mathbb{Q}$-linear map (called an evaluation map) $Z: \mathfrak{H}^{0} \longrightarrow \mathbb{R}$ by $Z(1)=1$ and

$$
Z\left(u_{1} u_{2} \cdots u_{k}\right)=\int_{1>t_{1}>t_{2}>\cdots>t_{k}>0} \cdots \int_{u_{1}}\left(t_{1}\right) \omega_{u_{2}}\left(t_{2}\right) \cdots \omega_{u_{k}}\left(t_{k}\right)
$$

$\left(u_{1}, u_{2}, \ldots, u_{k} \in\{x, y\}\right)$, where $\omega_{x}(t)=d t / t$ and $\omega_{y}(t)=d t /(1-t)$. As $u_{1} u_{2} \cdots u_{k}$ is in $\mathfrak{H}^{0}$, we always have $\omega_{u_{1}}(t)=d t / t$ and $\omega_{u_{k}}(t)=d t /(1-t)$, so the integral converges. By the Drinfel'd integral representation, we have

$$
Z\left(z_{k_{1}} z_{k_{2}} \cdots z_{k_{n}}\right)=\zeta\left(k_{1}, k_{2}, \ldots, k_{n}\right)
$$

We next define the shuffle product $\amalg$ on $\mathfrak{H}$ inductively by

$$
\begin{aligned}
1 ш w & =w \amalg 1=w, \\
u_{1} w_{1} \amalg u_{2} w_{2} & =u_{1}\left(w_{1} \amalg u_{2} w_{2}\right)+u_{2}\left(u_{1} w_{1} \amalg w_{2}\right)
\end{aligned}
$$

$\left(u_{1}, u_{2} \in\{x, y\}\right.$ and $w, w_{1}, w_{2}$ are words in $\left.\mathfrak{H}\right)$, together with $\mathbb{Q}$-bilinearity. The shuffle product $ш$ is commutative and associative. By the standard shuffle product identity of iterated integrals, the evaluation map $Z$ is a homomorphism with respect to the shuffle product $ш$ :

$$
Z\left(w_{1} ш w_{2}\right)=Z\left(w_{1}\right) Z\left(w_{2}\right) \quad\left(w_{1}, w_{2} \in \mathfrak{H}^{0}\right) .
$$

We also define the shuffle product $\widetilde{\mathrm{II}}$ on $\mathfrak{H}^{1}$ inductively by

$$
\begin{aligned}
1 \widetilde{\mathrm{\omega}} w & =w \widetilde{\mathrm{m}} 1=w, \\
u_{1} w_{1} \widetilde{\mathrm{\omega}} u_{2} w_{2} & =u_{1}\left(w_{1} \widetilde{\mathrm{\omega}} u_{2} w_{2}\right)+u_{2}\left(u_{1} w_{1} \widetilde{\mathrm{\omega}} w_{2}\right)
\end{aligned}
$$

$\left(u_{1}, u_{2} \in\left\{z_{k}\right\}_{k \geq 1}\right.$ and $w, w_{1}, w_{2}$ are words in $\left.\mathfrak{H}^{1}\right)$, together with $\mathbb{Q}$-bilinearity. For example, we have

$$
\begin{aligned}
z_{m} \widetilde{\amalg} z_{n} & =z_{m} z_{n}+z_{n} z_{m}, \\
z_{m} \widetilde{\amalg} z_{n} z_{l} & =z_{m} z_{n} z_{l}+z_{n} z_{m} z_{l}+z_{n} z_{l} z_{m} .
\end{aligned}
$$

Then Theorem 1 can be restated as follows:

$$
Z\left(z_{2}^{m} \widetilde{\mathrm{U}}\left(z_{3} z_{1}\right)^{n}\right)=\left(\begin{array}{c}
m+2 n \\
m
\end{array}\right) \frac{\pi^{2 m+4 n}}{(2 n+1) \cdot(2 m+4 n+1) !} \quad\left(m, n \in \mathbb{Z}_{\geq 0}\right) .
$$

\section{Proof of Theorem 1}

We restate Proposition 4.1 and Proposition 4.2 of 4 by using $\widetilde{\omega}$ and prove them by induction.

Proposition 2. For integers $n, N$ which satisfy $0 \leq n \leq N$, we have

$$
\begin{aligned}
& z_{2}^{n} \amalg z_{2}^{N}=\sum_{k=0}^{n} 4^{k}\left(\begin{array}{c}
N+n-2 k \\
n-k
\end{array}\right)\left\{z_{2}^{N+n-2 k} \widetilde{\amalg}\left(z_{3} z_{1}\right)^{k}\right\}, \\
& z_{1} z_{2}^{n} \amalg z_{1} z_{2}^{N}=2 \sum_{k=0}^{n} 4^{k}\left(\begin{array}{c}
N+n-2 k \\
n-k
\end{array}\right) z_{1}\left\{z_{2}^{N+n-2 k} \widetilde{\mathrm{\omega}} z_{1}\left(z_{3} z_{1}\right)^{k}\right\} .
\end{aligned}
$$


Proof. We prove identities (11) and (2) simultaneously by induction on $n$. [Step 1] The case $n=0$ of (1) is clear. We can easily prove the case $n=0$ of (2) by induction on $N$. [Step 2] Suppose that (11) and (2) have been proven for $n-1$. We prove (11) for $n$ by induction on $N$ :

$$
\begin{aligned}
z_{2}^{n} \amalg z_{2}^{n}= & 2 x y\left\{(x y)^{n-1} \amalg(x y)^{n}\right\}+2 x^{2}\left\{y(x y)^{n-1} \mathrm{\amalg} y(x y)^{n-1}\right\} \\
= & 2 \sum_{k=0}^{n-1} 4^{k}\left(\begin{array}{c}
2 n-1-2 k \\
n-1-k
\end{array}\right) z_{2}\left\{z_{2}^{2 n-1-2 k} \widetilde{\mathrm{\amalg}}\left(z_{3} z_{1}\right)^{k}\right\} \\
& +\sum_{k=0}^{n-1} 4^{k+1}\left(\begin{array}{c}
2 n-2-2 k \\
n-1-k
\end{array}\right) z_{3}\left\{z_{2}^{2 n-2-2 k} \widetilde{\mathrm{\omega}} z_{1}\left(z_{3} z_{1}\right)^{k}\right\} \\
= & \sum_{k=0}^{n-1} 4^{k}\left(\begin{array}{c}
2 n-2 k \\
n-k
\end{array}\right) z_{2}\left\{z_{2}^{2 n-1-2 k} \widetilde{\amalg}\left(z_{3} z_{1}\right)^{k}\right\} \\
& +\sum_{k=1}^{n} 4^{k}\left(\begin{array}{c}
2 n-2 k \\
n-k
\end{array}\right) z_{3}\left\{z_{2}^{2 n-2 k} \widetilde{\mathrm{\omega}} z_{1}\left(z_{3} z_{1}\right)^{k-1}\right\} \\
= & \left(\begin{array}{c}
2 n \\
n
\end{array}\right) z_{2}^{2 n}+\sum_{k=1}^{n-1} 4^{k}\left(\begin{array}{c}
2 n-2 k \\
n-k
\end{array}\right)\left\{z_{2}^{2 n-2 k} \widetilde{\mathrm{\amalg}}\left(z_{3} z_{1}\right)^{k}\right\}+4^{n}\left(z_{3} z_{1}\right)^{n} \\
= & \sum_{k=0}^{n} 4^{k}\left(\begin{array}{c}
2 n-2 k \\
n-k
\end{array}\right)\left\{z_{2}^{2 n-2 k} \widetilde{\mathrm{\omega}}\left(z_{3} z_{1}\right)^{k}\right\} .
\end{aligned}
$$

Hence (1) is true for $N=n$. Suppose that the case $N-1$ of (1) has been proven. (We may assume that $N-1 \geq n$ in the following calculation.)

$$
\begin{aligned}
& z_{2}^{n} \amalg z_{2}^{N}=x y\left\{(x y)^{n-1} \amalg(x y)^{N}\right\}+2 x^{2}\left\{y(x y)^{n-1} \amalg y(x y)^{N-1}\right\} \\
& +x y\left\{(x y)^{n} \amalg(x y)^{N-1}\right\} \\
& =\sum_{k=0}^{n-1} 4^{k}\left(\begin{array}{c}
N+n-1-2 k \\
n-1-k
\end{array}\right) z_{2}\left\{z_{2}^{N+n-1-2 k} \widetilde{\amalg}\left(z_{3} z_{1}\right)^{k}\right\} \\
& +\sum_{k=0}^{n-1} 4^{k+1}\left(\begin{array}{c}
N+n-2-2 k \\
n-1-k
\end{array}\right) z_{3}\left\{z_{2}^{N+n-2-2 k} \widetilde{\amalg} z_{1}\left(z_{3} z_{1}\right)^{k}\right\} \\
& +\sum_{k=0}^{n} 4^{k}\left(\begin{array}{c}
N+n-1-2 k \\
n-k
\end{array}\right) z_{2}\left\{z_{2}^{N+n-1-2 k} \widetilde{\mathrm{E}}\left(z_{3} z_{1}\right)^{k}\right\} \\
& =\sum_{k=0}^{n-1} 4^{k}\left(\begin{array}{c}
N+n-2 k \\
n-k
\end{array}\right) z_{2}\left\{z_{2}^{N+n-1-2 k} \widetilde{\amalg}\left(z_{3} z_{1}\right)^{k}\right\} \\
& +\sum_{k=1}^{n} 4^{k}\left(\begin{array}{c}
N+n-2 k \\
n-k
\end{array}\right) z_{3}\left\{z_{2}^{N+n-2 k} \widetilde{\mathrm{U}} z_{1}\left(z_{3} z_{1}\right)^{k-1}\right\} \\
& +4^{n} z_{2}\left\{z_{2}^{N-n-1} \widetilde{\mathrm{U}}\left(z_{3} z_{1}\right)^{n}\right\} \\
& =\left(\begin{array}{c}
N+n \\
n
\end{array}\right) z_{2}^{N+n}+\sum_{k=1}^{n-1} 4^{k}\left(\begin{array}{c}
N+n-2 k \\
n-k
\end{array}\right)\left\{z_{2}^{N+n-2 k} \widetilde{\mathrm{U}}\left(z_{3} z_{1}\right)^{k}\right\} \\
& +4^{n}\left\{z_{2}^{N-n} \widetilde{\mathrm{U}}\left(z_{3} z_{1}\right)^{n}\right\} \\
& =\sum_{k=0}^{n} 4^{k}\left(\begin{array}{c}
N+n-2 k \\
n-k
\end{array}\right)\left\{z_{2}^{N+n-2 k} \widetilde{\mathrm{W}}\left(z_{3} z_{1}\right)^{k}\right\} \text {. }
\end{aligned}
$$


Hence (11) is true for $N$. We can prove (2) for $n$ by induction on $N$ with using (1) for $n$.

Before proceeding to the proof of Theorem 1, we prove a key identity. Comparing coefficients of $(x+1)^{2 m+4 n+2}=\left(x^{2}+2 x+1\right)^{m+2 n+1}$, we have

$$
\left(\begin{array}{c}
2 m+4 n+2 \\
2 n+1
\end{array}\right)=\sum_{k=0}^{n} 2^{2 k+1} \frac{(m+2 n+1) !}{(n-k) !(2 k+1) !(m+n-k) !}
$$

We can transform this identity as follows:

$$
\begin{aligned}
& \frac{1}{(2 n+1) !} \frac{1}{(2 m+2 n+1) !} \\
& \quad=\sum_{k=0}^{n} 4^{k}\left(\begin{array}{c}
m+2 n-2 k \\
n-k
\end{array}\right)\left(\begin{array}{c}
m+2 n \\
2 k
\end{array}\right) \frac{1}{(2 k+1) \cdot(2 m+4 n+1) !} .
\end{aligned}
$$

Proof of Theorem 1. We prove Theorem 1 by induction on $n$. The case $n=0$ is well known as has been mentioned in Section 1. Suppose that the assertion has been proven up to $n-1$. Putting $N=m+n$ in (11), we have

$$
\begin{array}{rl}
4^{n} & Z\left(z_{2}^{m} \widetilde{\amalg}\left(z_{3} z_{1}\right)^{n}\right) \\
= & \frac{\pi^{2 n}}{(2 n+1) !} \frac{\pi^{2 m+2 n}}{(2 m+2 n+1) !} \\
& -\sum_{k=0}^{n-1} 4^{k}\left(\begin{array}{c}
m+2 n-2 k \\
n-k
\end{array}\right)\left(\begin{array}{c}
m+2 n \\
2 k
\end{array}\right) \frac{\pi^{2 m+4 n}}{(2 k+1) \cdot(2 m+4 n+1) !} \\
= & 4^{n}\left(\begin{array}{c}
m+2 n \\
m
\end{array}\right) \frac{\pi^{2 m+4 n}}{(2 n+1) \cdot(2 m+4 n+1) !} .
\end{array}
$$

In the first equality we have used the induction hypothesis and the formula $\zeta\left(\{2\}_{m}\right)$ $=\pi^{2 m} /(2 m+1)$ ! (the case $\left.n=0\right)$, and in the second equality we have used (3).

\section{REFERENCES}

[1] J. M. Borwein, D. M. Bradley, and D. J. Broadhurst, Evaluations of k-fold Euler/Zagier sums: A compendium of results for arbitrary $k$, Electron. J. Combin. 4, No. 2 (1997). MR 1444152 (98b:11091)

[2] J. M. Borwein, D. M. Bradley, D. J. Broadhurst and P. Lisonĕk, Combinatorial aspects of multiple zeta values, Electron. J. Combin. 5, Research Paper 38 (1998). MR.1637378 (99g:11100)

[3] J. M. Borwein, D. M. Bradley, D. J. Broadhurst and P. Lisonĕk, Special values of multiple polylogarithms, Trans. Amer. Math. Soc. 353, No. 3 (2001), 907-941. MR1709772 (2003i:33003)

[4] D. Bowman and D. M. Bradley, The algebra and combinatorics of shuffles and multiple zeta values, J. Combin. Theory Ser. A 97 (2002), 43-61. MR1879045 (2003j:05010)

[5] M. Hoffman, Multiple harmonic series, Pacific J. Math. 152 (1992), 275-290. MR.1141796 (92i:11089)

[6] M. Hoffman, The algebra of multiple harmonic series, J. Algebra 194 (1997), 477-495. MR.1467164(99e:11119) 
[7] K. Ihara, M. Kaneko, and D. Zagier, Derivation and double shuffle relations for multiple zeta values, Compos. Math. 142 (2006), 307-338. MR2218898(2007e:11110)

[8] D. Zagier, Values of zeta functions and their applications, First European Congress of Mathematics, Vol. II, Progr. Math., 120, Birkhäuser, Basel, 1994, pp. 497-512. MR1341859 $(96 \mathrm{k}: 11110)$

Graduate School of Mathematics, Kyushu University, Fukuoka 812-8581, Japan

E-mail address: muneta@math.kyushu-u.ac.jp 